\title{
A systematic review of euthyroid Graves' disease (Review)
}

\author{
MIOARA-LAURA MACOVEI*, ÜNAL AZIS*, \\ ALINA GABRIELA GHEORGHE and MARIAN BURCEA \\ Department of Ophthalmology, 'Carol Davila' University of Medicine and Pharmacy, \\ 050474 Bucharest, Romania
}

Received June 7, 2021; Accepted July 7, 2021

DOI: $10.3892 / \mathrm{etm} .2021 .10781$

\begin{abstract}
Euthyroid Graves' Disease (EGD) is a challenging pathology, due to its atypical clinical manifestations and the absence of abnormal thyroid function. Typically, thyroid ophthalmopathy is associated with elevated thyroid hormone levels and with the presence of thyrotropin receptor antibodies (TRAb) but a low percentage of patients remain euthyroid without developing hyperthyroidism during long-term follow-up periods. Although it is considered a different pathology, it shares a lot of similarities with Graves' disease, rendering the diagnosis more difficult. It is also important to note that ophthalmopathy may be the first clinical manifestation of Graves' disease and that thyroid function examinations do not present abnormalities over a long period. Treatment choices for euthyroid disease do not differ from those described in Graves' ophthalmopathy. However, it is considered that since euthyroid patients develop milder ophthalmic symptoms and their clinical activity score is lower, they tend to have better responses to treatment. Moreover, atypical sight-threatening cases such as exposure keratopathy and dysthyroid optic neuropathy with variable responses to therapy also exist. Disease management consists of a favorable collaboration between ophthalmologists and endocrinologists because patients with EGD can develop thyroid abnormalities over time. In conclusion, the diagnosis of EGD is difficult, clinical manifestations and evolution are variables depending on several factors, including the heterogeneity of TRAb. This review aimed to identify the characteristics of this disease by reviewing the clinical studies and case reports published in previous years.
\end{abstract}

Correspondence to: Dr Alina Gabriela Gheorghe, Department of Ophthalmology, 'Carol Davila' University of Medicine and Pharmacy, 8 Eroii Sanitari Boulevard, 050474 Bucharest, Romania E-mail: alina.gheorghe.g@gmail.com

*Contributed equally

Key words: Graves' disease, ophthalmopathy, euthyroid, hyperthyroid, thyrotropin receptor antibody

\section{Contents}

1. Methods

2. Introduction

3. Epidemiology and risk factors

4. Pathogenesis and diagnosis of EGD

5. Clinical characteristics of EGD

6. Treatment

7. Conclusions

\section{Methods}

A systematic review of literature regarding Euthyroid Graves' Disease (EGD) in Science Direct and PUBMED databases published since 2005 was conducted. The reviews which identified the characteristics of EGD were considered by comparing euthyroid, hypothyroid and hyperthyroid patients and case reports that presented unusual manifestations of EGD. The search revealed five reviews and thirteen case reports, and the studies analyzed were conducted in Asia, Europe, Latin and North America.

\section{Introduction}

Thyroid-associated ophthalmopathy (TAO) is the most common self-limited orbital inflammatory disorder associated with thyroid dysfunctions such as Graves' disease or Hashimoto thyroiditis (1). The systematic review of Muñoz-Ortiz et al revealed that the global prevalence in patients with thyroid-associated ophthalmopathy is $7.9 \%$ for euthyroidism, $10.36 \%$ for hypothyroidism, and $86.2 \%$ for hyperthyroidism (2). The onset of ophthalmopathy was reported to precede the thyroid dysfunction in $19.6 \%$ cases, while $80 \%$ of patients with Graves' disease had developed ophthalmologic symptoms within 18 months after the thyroid dysfunction (3).

Euthyroid Graves' disease (EGD) is a rare entity, which is defined as an infiltrative orbitopathy occurring in the absence of present or past thyroid clinical or biochemical abnormalities without any antithyroid treatment (4). Due to its low prevalence and incidence, the diagnosis is difficult to establish. A few reviews and case reports are available for further evaluation of clinical characteristics, course and treatment of this disease. Repeated testing of thyroid function is essential 
during the follow-up. Notably, euthyroid ophthalmopathy can represent the initial stage of thyroid dysfunction. It is also considered that a subset of patients with prior undetermined episodes of transient thyroid abnormalities is identified within the group of euthyroid patients (5). Long-term follow-up is essential in the management of EGD, and a retrospective study has highlighted that dysthyroidism could occur in $8-25 \%$ of patients between $15-45$ months from the initial presentation of ophthalmopathy (5). Furthermore, a patient with euthyroid ophthalmopathy was observed for twelve years without developing thyroid dysfunction (6).

\section{Epidemiology and risk factors}

It has been reported that the prevalence of Graves' orbitopathy (GO) with normal thyroid function varies between 0.9 and $15.4 \%$ (2). A comparative study between euthyroid and dysthyroid patients concluded that the average age of occurrence was 53.3 years in the euthyroid group and 54.6 in the dysthyroid group (7). Moreover, it was considered that the sex ratio was similar in the two groups, but Bhatnagar et al revealed female/male ratios of 1.62 and 3.2, indicating that euthyroid males presented more frequent orbital disease than females in the euthyroid group (7). Moreover, it was observed that euthyroid males develop milder asymmetric thyroid-associated ophthalmopathy (8).

Development of TAO is the result of the concurrence of several factors represented by endogenous triggers such as genetic factors, age, sex, high levels of thyrotropin receptor antibodies (TRAb), and environmental triggers: iodine intake, oxidative stress, cigarette smoking, psychosocial stress, bacterial and viral infections and certain medications such as interferon or alemtuzumab (2). Smoking prevalence in EGD is similar to hyper- and hypothyroid patients and it is strongly correlated with ophthalmopathy development and its severity; Termote et al identified that the smoking prevalence in EGD is $40 \%$ (5). It has been postulated that cigarette smoking is associated with TAO progression, increases the risk of development of proptosis and diplopia. It is also responsible for a decreased or delayed response to immunosuppressive treatments for moderate-to-severe, active GO (9).

It has been demonstrated that TRAb cause hyperthyroidism (10). The disease activity and clinical progression have been studied in euthyroid patients to obtain a correlation with thyroid stimulating antibody (TSAb) and thyrotropin binding inhibitor immunoglobulin (TBII) levels; Gerding et al assessed TSAb levels in a group of patients with untreated euthyroid ophthalmopathy. It has been determined that there is an important relationship between proptosis, the clinical activity of the disease and TSAb/TBII titers (11).

Inoue et al studied the genetic characteristics of euthyroid ophthalmopathy, by comparing HLA typing among patients with euthyroid ophthalmopathy and ophthalmopathy associated with Graves' disease or Hashimoto thyroiditis (12). The results in the three groups highlighted the unusual genetic pattern of euthyroid disease. The heterogeneity of EGD was also confirmed by important associations with the following antigens HLA B40 (w61), DR9, DQw3, HLA B12, and $\mathrm{Cw} 1$ (12). The most important difference between the three groups was observed in the HLA-B12 antigen, which revealed a 17-fold increase in euthyroid patients. Moreover, it has been suggested that persistent hyperthyroidism in EGD is prevented by several genetic factors. The rare incidence of family history of diffuse goiter in EGD patients increased the probability that the genetic factors have little effect on the development of ophthalmopathy (12). However, Ardley et al observed that genetic factors could play an important role, by reporting that $33 \%$ of euthyroid patients developed mild ocular symptoms associated with the presence of eye muscle antibodies against collagen XIII and CASQ1 (13).

\section{Pathogenesis and diagnosis of EGD}

Although EGD and Graves' disease share multiple similarities, they are different entities, suggested by the fact that the targets in EGD are represented by the eye and the orbit, while in Graves' disease it is the thyroid gland (2). Thyrotropin receptor (TSH-R) represents the common autoantigen for thyroid-associated ophthalmopathy and Graves' disease. Pathological changes in thyroid ophthalmopathy affect both extraocular muscles and orbital fat compartments. The molecular mechanisms behind thyroid ophthalmopathy consist of recruitment of $\mathrm{T}$ cells into the orbit, molecular interactions between immune system cells and orbital fibroblasts, which represent the effector cells leading to proliferation (14). Orbital fibroblasts are able to produce glycosaminoglycans and to differentiate into adipocytes and myofibroblasts. The proliferation of orbital fibroblasts is activated by the interaction of autoantigens on the fibroblasts with $\mathrm{T}$ cells. The synthesis of hyaluronan and the adipogenesis depend on the orbital fibroblast surface receptors such as TSH-R which induce activation of PI3K/Akt signaling cascade along with the adenylyl cyclase/cAMP pathway and insulin-like growth factor (IGF-1R) (14).

Furthermore, activated T cells infiltrate the orbit and secrete proinflammatory cytokines by interacting with autoreactive B cells. These cellular and molecular changes are responsible for extraocular muscle enlargement, orbital adipose tissue expansion, orbital inflammation, and tissue remodeling (15). Wakelkamp et al concluded that the level of TSH receptor mRNA was significantly higher in the adipose/connective tissue of active, untreated Graves' ophthalmopathy patients than in inactive patients (16). There appears to be an initial cellular autoimmune response that may be extended by a humoral autoimmune attack. The cytokine environment in the orbital fat tissues of these active Graves' ophthalmopathy patients consists mainly of T helper 1 (Th1) proinflammatory cytokines (16). A mechanism that involves calsequestrin derived from extraocular muscles as a target of autoimmunity has been described. It also represents a new sensitive and specific biomarker of ophthalmopathy in Graves' disease (17).

The diagnosis of EGD is often challenging and it is based on clinical aspects such as ophthalmopathy, which in EGD reveals a heterogeneous pattern, normal thyroid function examination (normal levels of thyroxine, tri-iodothyronine, and thyrotropin) without the prior existence of thyroid abnormalities, and also orbital imaging methods such as contrast-enhanced CT scan, T2/STIR MRI or ultrasonography. Positive serum thyroid antibodies such as TRAb, TBII, long-acting thyroid stimulator assays, thyroid-peroxidase antibodies (TPOAb) 
and thyroglobulin antibodies ( $\mathrm{TgAb}$ ) confirm the diagnosis (18). Both ophthalmopathy and thyroid-stimulating antibodies are specific indicators of Graves' disease. Kotwal and Stan suggested that in the atypical presentation of thyroid ophthalmopathy (absence of inflammatory signs or unilateral involvement), evaluating TRAb levels, by both TBII and TSAb bioassay would be helpful for the diagnosis of EGD (19). Other conditions such as idiopathic orbital inflammation, sarcoidosis, amyloidosis, cellulitis, orbital tumors or vascular malformations, vasculitis, lymphoma, drug-induced proptosis (lithium/corticosteroids) or hydrocephalus must be considered in the differential diagnosis and excluded (20).

\section{Clinical characteristics of EGD}

According to Rundle's curve, thyroid ophthalmopathy has a biphasic course, indicating that initially, there is an active, inflammatory, progressive phase, followed by a chronic, stable phase. The average duration of the active phase is between 6-18 months (21). The most common symptoms of thyroid-associated ophthalmopathy include eyelid retraction, proptosis, restrictive strabismus and dysthyroid optic neuropathy (15). Ptosis in euthyroid ophthalmopathy is indicative of the association with myasthenia gravis (22). Several clinical studies and case reports were analyzed in order to determine the characteristics of EGD. A comparison between patients with EGD and medically untreated patients with Graves' ophthalmopathy was performed by Komoto et al. This 3 -year study concluded that EGD is more common among males; ocular findings such as proptosis, retraction of the lid and enlargement of extraocular muscles were more frequent among EGD patients (23). Regarding the thyroid function and antibodies, thyrotropin levels were higher in EGD patients, while TSAb levels in EGD were similar to another report (23). It was highlighted that TSAb was present in $40 \%$ of cases, while TRAb, TPOAb, and TgAb were identified in 35, 25 and $21 \%$ of cases (23).

Eckstein et al studied the prevalence, clinical severity and activity, antibody levels and the course of the disease among hyperthyroid, hypothyroid and euthyroid Caucasian patients with Graves' ophthalmopathy (24). The follow-up period was 36 months, including ophthalmic assessment and thyroid function examination. The severity of ophthalmopathy was determined by using NO SPECS criteria, while the activity was assessed by Mourits' Clinical Activity Score (CAS). The results of this study confirmed that cigarette smoking represents an important risk factor for developing ophthalmopathy, having a similar percentage in all three groups. Euthyroid patients had developed milder symptoms with lower activity scores. Soft-tissue inflammation was less present in the euthyroid group. The ophthalmopathy developed asymmetrically, with a proptosis difference of $>3 \mathrm{~mm}$ (24). The increased frequency of asymmetry was also observed in numerous euthyroid patients by Kavoussi et al (8). Neither developed severe sight loss, nor dysthyroid optic neuropathy. Endocrinological findings indicated that levels of TRAb were lower in the euthyroid group at the 6-month follow-up. The most important predictive factors of euthyroid ophthalmopathy are represented by suppressed TSH and the presence of TRAb. It has been suggested that the diagnosis of the euthyroid disease is difficult due to asymmetrical manifestations and due to the existence of a temporal relationship between the onset of thyroid dysfunction and ophthalmopathy (25). Approximately $18 \%$ of patients with Graves' disease tend to develop thyroid dysfunction in a year after the onset of the ophthalmopathy, while $25 \%$ of euthyroid patients are expected to exhibit thyroid abnormalities within 4 years. It was also reported that the assessment of TSAb is more sensitive and also a useful tool for diagnosis of euthyroid ophthalmopathy (24).

Another clinical study conducted by Jang et al among Korean euthyroid patients revealed that unilateral involvement is more frequently observed in euthyroid patients (79.2\%). Upper eyelid retraction was present in $91.7 \%$ of patients (3). The activity and severity of euthyroid patients were similar to those studied by Eckstein et al (24). The average duration of ophthalmic symptoms was 3 months and it was suggested that it had a great influence on clinical activity or severity. Thyroid function was maintained normal in most of the cases, although a few patients developed abnormalities. TRAb represented an important diagnosis marker but there were lower levels in euthyroid patients (3). Moreover, a few cases developed ophthalmopathy without the presence of TRAb; it was reported that TRAb levels vary over time and the importance of TRAb assessments as early as possible in the presence of typical ocular signs and symptoms was underlined. It was suggested that TSAb assessments were more specific than TBII assays (26).

In a clinical study conducted by Termote $\mathrm{et} \mathrm{al}$, the euthyroid patients developed milder and unilateral ophthalmopathy (5). The discovery was that previous exposure to high levels of thyroid hormone in euthyroid ophthalmopathy could be associated with proptosis and extraocular muscle restriction (5). It was also revealed that normal thyroid function influences the course of the GO positively (27). Khoo et al (28) observed that the elevated TRAb levels are involved in the development of hyperthyroidism, while Termote concluded that TRAb levels were more elevated in EGD patients with a history of thyroid hyperfunction. The importance of long-term follow-up in EGD patients was also highlighted, due to the fact that ophthalmopathy could be the initial manifestation of Graves' disease (28).

The mechanisms involved in the normal thyroid function of euthyroid patients are unclear. It has been suggested that the low levels and activity of TSAb cannot determine hyperthyroidism in EGD patients. Furthermore, a suppressive mechanism against TSAb may exist. Moreover, the antigenic epitopes identified on TSH receptors of EGD patients revealed differences compared with those identified in hyperthyroid Graves' disease (29). The thyroid function should be evaluated during the follow-up. It was observed that euthyroid patients may develop thyrotoxicosis (30) or hypothyroidism (31) over time. The abnormalities upon thyroid examination identified among euthyroid patients are the presence of thyroid enlargement (24-40\%), low TSH (14-23\%), no or low response of TSH to thyrotropin releasing hormone (TRH) stimulation (40-63\%), high thyroid uptake of iodine (20\%), negative TSH suppression test (30-73\%), positive $\operatorname{Tg} \mathrm{Ab}$ (11-67\%), positive TBII (31-36\%) and positive TSAb (43-87\%) (29). Several cases with negative autoantibodies in patients with specific signs of ophthalmopathy have been reported $(26,32,33)$. Moreover, Watanabe et al 
Table I. Comparison of clinical characteristics between ophthalmopathy associated with EGD and hyperthyroid Graves' disease.

\begin{tabular}{|c|c|c|}
\hline Clinical characteristics & EGD & $\begin{array}{l}\text { Hyperthyroid } \\
\text { Graves' disease }\end{array}$ \\
\hline Age & Similar & Similar \\
\hline Smoker proportion & Similar & Similar \\
\hline $\begin{array}{l}\text { Duration of ophthalmic } \\
\text { symptoms }\end{array}$ & Similar & Similar \\
\hline Male/female ratio & More frequent in men & \\
\hline $\begin{array}{l}\text { Unilateral Graves' } \\
\text { ophthalmopathy }\end{array}$ & More often reported & \\
\hline Clinical activity score & Mild/ moderate cases & $\begin{array}{l}\text { Moderate-severe/ } \\
\text { sight-threatening cases }\end{array}$ \\
\hline NO SPECS & Less active & \\
\hline Soft-tissue inflammation & More prevalent & \\
\hline Lid retraction & More prevalent & \\
\hline Optic neuropathy & & More frequent \\
\hline Restrictive myopathy & & More frequent \\
\hline Average Hertel values & Similar & Similar \\
\hline Proptosis $>3 \mathrm{~mm}$ & More prevalent & \\
\hline Steroid therapy & Similar & Similar \\
\hline Orbital irradiation & & More frequent \\
\hline
\end{tabular}

EGD, Euthyroid Graves' Disease; NO SPECS, classification of ophthalmic changes in Graves' ophthalmopathy (class 0 , no signs or symptoms; class 1 , only signs no symptoms; class 2 , soft tissue involvement; class 3, proptosis; class 4, extraocular muscle involvement; class 5, corneal involvement; class 6, sight loss).

identified the presence of TSAb in $93 \%$ of cases by evaluation with a sensitive bioassay, which indicated its usefulness in the diagnosis of EGD with negative-autoantibodies. The activity of TSAb is also correlated with the extent of the proptosis, indicating that TSAb is an important tool in ophthalmopathy development by interacting with TSH receptors found in orbital tissue (29).

Although euthyroid ophthalmopathy is milder and more asymmetrical, it should be noted that exposure keratopathy (34) or optic neuropathy (35) could develop during the early stages as the initial manifestation. Several cases of euthyroid optic neuropathy have been reported. Lee et al described a case of bilateral euthyroid optic neuropathy with severe vision loss, despite intravenous corticosteroid treatment (36). In addition, two case reports presented unilateral optic neuropathy as the initial clinical manifestation of euthyroid ophthalmopathy. In both cases, vision loss was improved, restoring the initial visual acuity and visual field. In the first case corticosteroid therapy was administered (35), while the second case was only observed (37). A conservatory strategy can be rational in cases where disease severity has already peaked and clinical activity score is low; the authors concluded that the spontaneous remission of optic neuropathy can be correlated with normal thyroid function and with the absence of thyroid antibodies (37).

Orbital imagistic techniques revealed tendon-sparing enlargement of lateral and medial recti (3). Dickinson and Perros suggested that the inferior rectus is most frequently involved in thyroid-associated ophthalmopathy, followed by medial, superior, and lateral rectus (38). The involvement of the inferior oblique muscle in EGD in a 38-year old female, presenting unilateral blepharoptosis, intraocular pain and headache was also reported (39).

Tables I and II aimed to compare the clinical and endocrinological differences of ophthalmopathy associated with EGD and Graves' disease.

\section{Treatment}

The management of EGD depends on the scale of severity EUGOGO as a mild, moderate-severe, and sight-threatening disease. Moreover, the clinical activity score has an important role in treatment choice (40).

Treatment for euthyroid Graves' disease in the active phase implies anti-inflammatory therapies which reduce the immune response and promote immune system modulation (41). It has been revealed in several studies and reports that the prognosis is usually favorable and surgical approach is rare. In all cases, cessation of smoking has revealed an important role in relieving symptoms, the clinical course of the disease, and response to treatment (40-44).

Most patients with mild ophthalmopathy can be observed; the follow-up interval is related to disease activity. In moderate to severe active Graves' ophthalmopathy cases, intravenous corticosteroid therapy combined with orbital radiation therapy can improve symptoms as lid swelling or proptosis but are inefficient for diplopia. Glucocorticoids have also demonstrated satisfactory results in TRAb-negative euthyroid 
Table II. Thyroid function examinations and autoantibodies in EGD vs. hyperthyroid Graves' disease.

\begin{tabular}{lcc}
\hline $\begin{array}{l}\text { Thyroid function examinations } \\
\text { and autoantibodies }\end{array}$ & EGD & $\begin{array}{c}\text { Hyperthyroid } \\
\text { Graves' disease }\end{array}$ \\
\hline $\begin{array}{l}\text { Serum fT4 } \\
\text { Serum T3 }\end{array}$ & Normal & Normal/Elevated \\
TSH & Normal & Normal/Elevated \\
TRAb at first presentation & Normal & Decreased \\
TBII levels 6 months after & $+/-$ & Lower \\
the onset of GO symptoms & & Higher \\
TPO Ab 6 months after the & Similar & Similar
\end{tabular}

EGD, Euthyroid Graves' Disease; fT4, thyroxine; T3, tri-iodothyronine; TSH, thyroid stimulating hormone/thyrotropin; TRAb, thyroid stimulating hormone receptor antibodies; TBII, thyrotropin-binding inhibitor immunoglobulin; TPO Ab, thyroid peroxidase antibody; GO, Graves' ophthalmopathy.

ophthalmopathy cases. Surgical rehabilitation interventions on extraocular muscles are recommended in the inactive phase and may relieve double vision or strabismus. Blepharoplasties can also be performed (23).

Severe sight-threatening euthyroid optic neuropathy cases were also reported by authors. The-first line therapy in these patients is represented by intravenously administered steroids, followed by orbital decompression surgery in irresponsive cases. It was suggested that the clinical response to initial corticosteroid therapy has variable effectiveness. The efficiency of pulse corticosteroid treatment is assessed by visual acuity recovery, which was described especially in cases with unilateral involvement (36). Atypical presentation of euthyroid optic neuropathy with bilateral involvement and severe deterioration in visual acuity revealed a slight amelioration after the intravenous corticosteroid therapy (37).

Suzuki et al reported that $50 \%$ of the EGD patients included in their clinical study received ophthalmic treatment as follows: $62 \%$ of patients required local injection of triamcinolone, $55.2 \%$ radiotherapy in monotherapy or combined with glucocorticoids and $20.6 \%$ underwent different surgical procedures such as orbital decompression or strabismus surgery (45). Most of the symptoms of thyroid-associated ophthalmopathy improved, although a few patients relapsed over three years and received intravenous glucocorticoids, local steroid injections or they were eligible for surgical treatment. Half of the patients exhibited spontaneous improvement without any treatment at their first assessment (45).

There is no evidence of different management of euthyroid ophthalmopathy but it may be useful to consider the efficacy of novel therapies in this group of cases. Two different studies on teprotumumab and rituximab on monoclonal antibodies, represented by clinical trials compared the results in patients with ophthalmopathy and treatment-induced euthyroidism with control patients $(45,46)$. The new agents are important in high-dose steroid unresponsive cases, exhibiting little adverse effects. A human monoclonal antibody, an inhibitor of IGF-1 receptor, teprotumumab has been recently approved for the treatment of active thyroid ophthalmopathy. Douglas et al revealed that this novel medication has some beneficial effects on proptosis and diplopia, reduces the CAS and improves the quality of life. Due to the fact that severe adverse effects are rare, teprotumumab could be considered an alternative to surgical treatment in TAO (46). Rituximab represents a chimeric humanized anti-CD20 which targets B cells and determines a decrease in antibody production which leads to the interruption of the activation of TSH-R found on the surface of orbital fibroblasts. It is considered that rituximab reduces CAS score and proptosis (47).

The treatment of thyroid ophthalmopathy should have a multidisciplinary approach including oculoplastic surgery, strabismus surgery, and neuro-ophthalmology with a periodic thyroid profile assessment in order to manage the symptoms which can develop during the evolution of this disease (48).

\section{Conclusions}

Ophthalmologists should be aware of EGD, which is a rare entity with an atypical initial presentation. Although there are studies on the pathogenesis, clinical manifestations, evolution and treatment, in most of the cases the diagnosis is difficult. The asymmetrical, mild pattern of ophthalmopathy and normal thyroid examination function are usually indicative of EGD. Thus, it is confirmed by assessing specific autoantibodies. Patients with indicative signs and symptoms must be evaluated for thyroid ophthalmopathy, even though autoantibodies are negative at the initial presentation. The follow-up interval should be larger since it may be the primary manifestation of Graves' disease and thyroid abnormalities may appear several months after the onset of ophthalmopathy.

\section{Acknowledgements}

Not applicable.

\section{Funding}

No funding was received. 


\section{Availability of data and materials}

Not applicable.

\section{Authors' contributions}

MLM and ÜA conceived the study, performed the relevant literature research and prepared the manuscript. AGG and MB participated in the entire review process and contributed to the analysis and critical interpretation of the data. All authors read and approved the final manuscript.

\section{Ethics approval and consent to participate}

Not applicable.

\section{Patient consent for publication}

Not applicable.

\section{Competing interests}

The authors declare that they have no competing interests.

\section{References}

1. Wang Y and Smith TJ: Current concepts in the molecular pathogenesis of thyroid-associated ophthalmopathy. Invest Ophthalmol Vis Sci 55: 1735-1748, 2014.

2. Muñoz-Ortiz J, Sierra-Cote MC, Zapata-Bravo E, ValenzuelaVallejo L, Marin-Noriega MA, Uribe-Reina P, Terreros-Dorado JP, Gómez-Suarez M,Arteaga-Rivera K and de-la-Torre A: Prevalence of hyperthyroidism, hypothyroidism, and euthyroidism in thyroid eye disease: A systematic review of the literature. Syst Rev 9: 201, 2020.

3. Jang SY, Lee SY, Lee EJ and Yoon JS: Clinical features of thyroid-associated ophthalmopathy in clinically euthyroid Korean patients. Eye (Lond) 26: 1263-1269, 2012.

4. Boddu N, Jumani M, Wadhwa V, Bajaj G and Faas F: Not all orbitopathy is Graves': Discussion of cases and review of literature. Front Endocrinol (Lausanne) 8: 184, 2017.

5. Termote K, Decallonne B and Mombaerts I: The influence of prior hyperthyroidism on euthyroid graves' ophthalmopathy. J Ophthalmol 2014: 426898, 2014.

6. Peter SA: Euthyroid Graves' disease. Report of a case observed over a 12-year period. Am J Med 80: 1197-1198, 1986.

7. Bhatnagar A, Tsirbas A, Douglas RS, Goldberg RA and Hoyama E: Graves' orbitopathy. Ophthalmology 114: 392.e1-e2, 2007.

8. Kavoussi SC, Giacometti JN, Javier Servat JJ and Levin F: The relationship between sex and symmetry in thyroid eye disease. Clin Ophthalmol 8: 1295-1300, 2014.

9. Bartalena L and Piantanida E: Cigarette smoking: Number one enemy for Graves ophthalmopathy. Pol Arch Med Wewn 126: 725-726, 2016.

10. Takasu N, Oshiro C, Akamine H, Komiya I, Nagata A, Sato Y, Yoshimura $\mathrm{H}$ and Ito $\mathrm{K}$ : Thyroid-stimulating antibody and TSH-binding inhibitor immunoglobulin in 277 Graves' patients and in 686 normal subjects. J Endocrinol Invest 20: 452-461, 1997.

11. Gerding MN, Van Der Meer JW, Broenink M, Bakker O, Wiersinga WM and Prummel MF: Association of thyrotrophin receptor antibodies with the clinical features of Graves' ophthalmopathy. Clin Endocrinol (Oxf) 52: 267-271, 2000.

12. Inoue D, Sato K, Maeda M, Inoko H, Tsuji K, Mori T and Imura H: Genetic differences shown by HLA typing among Japanese patients with euthyroid Graves' ophthalmopathy, Graves' disease and Hashimoto's thyroiditis: Genetic characteristics of euthyroid Graves' ophthalmopathy. Clin Endocrinol (Oxf) 34: 57-62, 1991.

13. Ardley M, McCorquodale T, Lahooti H, Champion B and Wall JR: Eye findings and immunological markers in probands and their euthyroid relatives from a single family with multiple cases of thyroid autoimmunity. Thyroid Res 5: 4, 2012.
14. Khoo TK and Bahn RS: Pathogenesis of Graves' ophthalmopathy: The role of autoantibodies. Thyroid 17: 1013-1018, 2007.

15. Stan MN, Garrity JA and Bahn RS: The evaluation and treatment of Graves ophthalmopathy. Med Clin North Am 96: 311-328, 2012.

16. Wakelkamp IM, Bakker O, Baldeschi L, Wiersinga WM and Prummel MF: TSH-R expression and cytokine profile in orbital tissue of active vs inactive Graves' ophthalmopathy patients. Clin Endocrinol (Oxf) 58: 280-287, 2003.

17. Gopinath B, Musselman R, Beard N, El-Kaissi S, Tani J, Adams CL and Wall JR: Antibodies targeting the calcium binding skeletal muscle protein calsequestrin are specific markers of ophthalmopathy and sensitive indicators of ocular myopathy in patients with Graves' disease. Clin Exp Immunol 145: 56-62, 2006.

18. Krishnan VM, Baba D, Kumar KR and Venkatesh P: Case report a case of unilateral euthyroid Graves' disease. Sch J App Med Sci 3: 2534-2536, 2015.

19. Kotwal A and Stan M: Thyrotropin receptor antibodies-an overview. Ophthal Plast Reconstr Surg 34 (Suppl 1): S20-S27, 2018.

20. Bartley GB and Gorman CA: Diagnostic criteria for Graves ophthalmopathy. Am J Ophthalmol 119: 792-795, 1995.

21. Bartley G: Rundle and His Curve. Arch Ophthalmol 129: 356-358, 2011.

22. Marinò M, Barbesino G, Pinchera A, Manetti L, Ricciardi R, Rossi B, Muratorio A, Braverman LE, Mariotti S and Chiovato L: Increased frequency of euthyroid ophthalmopathy in patients with Graves' disease associated with myasthenia gravis. Thyroid 10: 799-802, 2000.

23. Komoto N, Kozaki A, Yu F, Inoue R, Maeda T, Inoue $\mathrm{T}$ and Inoue Y: Clinical feature of euthyroid Graves' ophthalmopathy. Invest Ophthalmol Vis Sci 49: 5250, 2008.

24. Eckstein AK, Lösch C, Glowacka D, Schott M, Mann K, Esser J and Morgenthaler NG: Euthyroid and primarily hypothyroid patients develop milder and significantly more asymmetrical Graves ophthalmopathy. Br J Ophthalmol 93: 1052-1056, 2009.

25. Wiersinga WM, Smit T, van der Gaag R and Koornneef L: Temporal relationship between onset of Graves' ophthalmopathy and onset of thyroidal Graves' disease. J Endocrinol Invest 11: 615-619, 1988.

26. Cakir M: Euthyroid Graves' ophthalmopathy with negative autoantibodies. J Natl Med Assoc 97: 1547-1549, 2005.

27. Hegedüs L, Bonnema SJ, Smith TJ and Brix TH: Treating the thyroid in the presence of Graves' ophthalmopathy. Best Pract Res Clin Endocrinol Metab 26: 313-324, 2012.

28. Khoo DH, Eng PH, Ho SC, Tai ES, Morgenthaler NG, Seah LL, Fong KS, Chee SP, Choo CT and Aw SE: Graves' ophthalmopathy in the absence of elevated free thyroxine and triiodothyronine levels: Prevalence, natural history, and thyrotropin receptor antibody levels. Thyroid 10: 1093-1100, 2000.

29. Watanabe M, Iwatani Y, Kashiwai T, Iijima T, Fujikado T and Amino N: Euthyroid Graves' disease showing no thyroid abnormalities except positive thyroid-sstimulating antibody (TSAb): Two case reports. J Intern Med 238: 379-384, 1995.

30. Tanaka K, Nomura T, Ohta Y, et al: A case of euthyroid Graves disease which developed into Graves disease with thyrotoxicosis after subacute thyroiditis. The Endocrinologist 19: 124-125, 2009.

31. Majumder A and Chatterjee S: Euthyroid Graves' ophthalmopathy patients remaining stable on more than 20 months follow up-a report of 9 patients. Hot Thyroidol, 2019.

32. Hotta A, Tanaka T, Kato H, Kakoi S, Shimizu Y, Hasegawa C, Hayakawa A, Yasuda S, Ogawa K, Ito S, et al: A case of euthyroid Graves' ophthalmopathy in a patient sero-negative for TSH receptor autoantibody. Case Rep Endocrinol 2018: 1707959, 2018.

33. Tabasum A, Khan I, Taylor P, Das G and Okosieme OE: Thyroid antibody-negative euthyroid Graves' ophthalmopathy. Endocrinol Diabetes Metab Case Rep 2016: 160008, 2016.

34. Shah Y: Thyroid ophthalmopathy. J Assoc Physicians India 59 (Suppl 1): S60-S65, 2011.

35. Arslan E, Yavaşoğlu I, Çildağ BM and Kocatürk T: Unilateral optic neuropathy as the initial manifestation of euthyroid Graves' disease. Intern Med 48: 1993-1994, 2009.

36. Lee YC, Chang FL and Tsai RK: Intravenous pulse corticosteroid therapy in euthyroid optic neuropathy: A case report. Neuro Ophthalmol 36: 143-145, 2012.

37. Kitaguchi Y, Ishihara K and Nishida K: Spontaneous resolution of euthyroid optic neuropathy. Can J Ophthalmol 54: e188-e192, 2019.

38. Dickinson AJ and Perros P: Controversies in the clinical evaluation of active thyroid-associated orbitopathy: Use of a detailed protocol with comparative photographs for objective assessment. Clin Endocrinol (Oxf) 55: 283-303, 2001. 
39. Kakizaki H, Zako $\mathrm{M}$ and Iwaki M: Thyroid-associated inferior oblique myopathy. Ophthalmology 114: 2106, 2007.

40. Bartalena L, Baldeschi L, Boboridis K, Eckstein A, Kahaly GJ, Marcocci C, Perros P, Salvi M and Wiersinga WM; European Group on Graves' Orbitopathy (EUGOGO): The 2016 European thyroid association/European group on Graves' orbitopathy guidelines for the management of Graves' orbitopathy. Eur Thyroid J 5: 9-26, 2016.

41. Wawhal M, Mogal V, Patil P, Ahire P and Yadav S: Euthyroid ophthalmopathy: A rare case report and a brief review of literature. Int J Sci Res 4: 529-532, 2015.

42. Ursu HI, Hortopan D, Podia-Igna C, Vizireanu A, Harsan T, Dumitriu L and Alexandrescu D: Late onset Graves' thyrotoxicosis in a patient with 18 years history of euthyroid Graves' ophthalmopathy. Acta Endo (Buc) 1: 227-232, 2005.

43. Sarkar PK, Sarkar P, Acharjee U, Halder S, Sarkar S and Noatia C: Graves ophthalmopathy in a euthyroid patient-a rare case report. J Evol Med Dent Sci 3: 7150-7155, 2014.

44. Khan AA, Mahmoud Muammar AR and Kamal I: A case of unilateral exophthalmos due to thyroid orbitopathy in a clinically euthyroid patient. World J Res Rev 9: 1-3, 2019.
45. Suzuki N, Noh JY, Kameda T, Yoshihara A, Ohye H, Suzuki M, Matsumoto M, Kunii Y, Iwaku K, Watanabe N, et al: Clinical course of thyroid function and thyroid associated-ophthalmopathy in patients with euthyroid Graves' disease. Clin Ophthalmol 12: 739-746, 2018.

46. Douglas RS, Kahaly GJ, Patel A, Sile S, Thompson EHZ, Perdok R, Fleming JC, Fowler BT, Marcocci C, Marinò M, et al: Teprotumumab for the treatment of active thyroid eye disease. $\mathrm{N}$ Engl J Med 382: 341-352, 2020.

47. Stan MN, Garrity JA, Carranza Leon BG, Prabin T, Bradley EA and Bahn RS: Randomized controlled trial of rituximab in patients with Graves' orbitopathy. J Clin Endocrinol Metab 100: 432-441, 2015.

48. Hodgson NM and Rajaii F: Current understanding of the progression and management of thyroid associated orbitopathy: A systematic review. Ophthalmol Ther 9: 21-33, 2020.

(F) (9) This work is licensed under a Creative Commons EY No No Attribution-NonCommercial-NoDerivatives 4.0 International (CC BY-NC-ND 4.0) License. 